\title{
Influência de aditivos dispersantes e acelerador na hidratação de cimento e cimento-matriz
}

\section{(Influence of dispersant and accelerator additives on hydration of calcium aluminate cement and cement-matrix)}

\author{
I. R. Oliveira, J. R. Garcia, V. C. Pandolfelli \\ Grupo de Engenharia de Microestrutura de Materiais - GEMM \\ Departamento de Engenharia de Materiais, Universidade Federal de S. Carlos \\ Rod. Washington Luiz, km 235, C.P. 676, S. Carlos, SP, 13565-905 \\ ivone@iris.ufscar.br,vicpando@power.ufscar.br
}

\begin{abstract}
Resumo
A aplicação de concretos refratários, principalmente na siderurgia, é um processo em constante evolução, que apresenta uma forte dependência dos avanços dos conhecimentos sobre os ligantes hidráulicos. Isso se deve a influência exercida por estes ligantes nas propriedades reológicas e desenvolvimento de resistência mecânica de concretos. O processo de hidratação dos ligantes é sensivelmente influenciado pela presença de aditivos, afetando o tempo requerido para efetuar a desmoldagem do corpo conformado. Além disso, a adição de alumina ao cimento também influencia o seu comportamento de hidratação, bem como a extensão do período de indução, a composição das fases e dos produtos de hidratação. Assim, neste trabalho foi estudada a influência da presença de aditivos dispersantes e/ou acelerador no processo de hidratação de cimento e cimento-matriz. Independente do sistema, os aditivos dispersantes atuaram como retardadores do processo de hidratação, principalmente para o caso do ácido cítrico e citrato de diamônio. Tais aditivos apresentaram-se também como os mais eficientes para a combinação com o acelerador $\mathrm{Li}_{2} \mathrm{CO}_{3}$, resultando em um tempo de pega intermediário. Isto mostra ser possível controlar a trabalhabilidade reduzindo o tempo para a desmoldagem. Palavras-chave: hidratação, aluminato de cálcio, dispersantes, matriz, acelerador.
\end{abstract}

\begin{abstract}
A growing demand for refractory castables with specific behaviors has been promoting a continuous technological evolution, in which, one of the most important aspects concerns in a deep knowledge of hydraulic binders. These materials present a great influence on the rheological properties and mechanical strength development of castables, defining their workability periods and demoulding times, respectively. The hydration process of calcium aluminate cement is influenced by the presence of additives, which affects the setting and demoulding time of the shaped body. Besides that, the alumina addition to cement also influences the hydration behaviour, as well as, the length of the induction period, the composition of the phases and of the hydration products. Therefore, in this work it was studied the influence of the dispersant and/or accelerator additives on the hydration process of calcium aluminate cement and cement-matrix. Independent of the system, the dispersant additives acted as retarders on hydration process, mainly the citric acid and diammonium citrate. These additives were the most efficient to combine with the accelerator $\left(\mathrm{Li}_{2} \mathrm{CO}_{3}\right)$ resulting an intermediate setting time, good workability and a short demolding time.
\end{abstract}

Keywords: hydration, calcium aluminate cement, dispersants, matrix, accelerator.

\section{INTRODUÇÃO}

O estado de dispersão da matriz do concreto apresenta uma grande influência no comportamento reológico desse material, determinando os métodos utilizados para a sua aplicação. Esforços têm se concentrado no desenvolvimento de métodos que possibilitem a automação dessa etapa e possam minimizar a incorporação de defeitos ao revestimento, melhorando a confiabilidade e as propriedades finais do refratário. Para tal aplicação normalmente é exigida a preparação de concretos com elevada fluidez, que possam ser bombeados com facilidade e sejam capazes de preencher moldes de formato complexo sem a necessidade de aplicação de vibração. Isso pode ser obtido por meio da adição de aditivos dispersantes favorecendo uma boa trabalhabilidade do concreto. Entretanto, tais aditivos também apresentam influência sobre o tempo requerido para efetuar a desmoldagem do corpo conformado $[1,2]$.

A resistência mecânica à verde do concreto está intimamente ligada ao processo de hidratação do ligante 
hidráulico (cimentos de alta alumina) [3]. Tal processo resulta na formação de uma estrutura que interliga as diferentes regiões da matriz do concreto e dos agregados, mantendo o material coeso. Uma vez acelerado este processo de hidratação, o desenvolvimento da resistência mecânica ocorrerá com maior rapidez, reduzindo o tempo para a desmoldagem do concreto [4].

A fase CA é a principal componente dos cimentos de alta alumina e tem uma cinética de hidratação relativamente lenta. Já a fase $\mathrm{C}_{12} \mathrm{~A}_{7}$, apresenta um tempo muito curto para o início da pega. Fases com menor razão $\mathrm{CaO}: \mathrm{Al}_{2} \mathrm{O}_{3}$, como o $\mathrm{CA}_{2}$, são consideravelmente menos reativas, no entanto, mais refratárias. A cinética de hidratação das fases do cimento pode ser bastante diminuída se baixas temperaturas de cura são aplicadas, resultando em tempos de pega mais longos para se obter a total hidratação [5].

$\mathrm{O}$ processo de hidratação do cimento inicia-se quando a água entra em contato com a superfície das partículas do cimento, sendo esta etapa descrita por meio da seguinte equação química:

$$
\mathrm{Ca}\left(\mathrm{AlO}_{2}\right)_{2}+4 \mathrm{H}_{2} \mathrm{O} \Leftrightarrow \mathrm{Ca}^{2+}+2 \mathrm{Al}(\mathrm{OH})_{4}^{-}
$$

Os íons $\mathrm{Al}(\mathrm{OH})_{4}^{-}$comportam-se como uma base, sendo que uma pequena parte deles se dissocia em íons $\mathrm{Al}^{3+} \mathrm{e} \mathrm{OH}^{-}$ em solução, estabelecendo um equilíbrio comandado pela constante básica $\mathrm{K}_{\mathrm{b}}$. Este fenômeno é o responsável pelo aumento do $\mathrm{pH}$ do meio:

$$
\mathrm{Al}(\mathrm{OH})_{4}^{-} \Leftrightarrow \mathrm{Al}^{3+}+4 \mathrm{OH}^{-} \quad \mathrm{K}_{\mathrm{b}}=1,8 \times 10^{2}
$$

A ocorrência destas reações químicas provoca a dissolução das fases anidras que compõem o cimento proporcionando um aumento das concentrações dos íons $\mathrm{Ca}^{2+}$ e $\mathrm{Al}(\mathrm{OH})_{4}^{-}$em solução. Este processo continua até que um estado de saturação da solução, em relação à dissolução do cimento, seja alcançado. Desta forma, o processo atinge um estado de equilíbrio químico e a dissolução do cimento é interrompida. Entretanto, em relação aos produtos da reação de hidratação, o equilíbrio alcançado não é estável, ou seja, a solução está supersaturada em relação aos compostos formadose, por esse motivo, existe uma tendência natural para que ocorra a precipitação desses produtos [5].

Apesar de favorecida, a precipitação dos hidratos envolve um processo lento de nucleação, durante o qual não ocorre precipitação. Esse tempo de espera para que se atinja condições ideais para a precipitação é conhecido como "período de indução". Este estado de equilíbrio metaestável é mantido até que se vença a barreira de energia de ativação $\left(\mathrm{E}_{\mathrm{a}}\right)$ necessária à formação dos primeiros germes dos cristais dos hidratos [5].

A partir da formação destes germes de hidratos a velocidade do processo não é mais limitada pela $\mathrm{E}_{\mathrm{a}}$, uma vez que a precipitação passa a ocorrer por meio do crescimento deles. Dessa maneira, íons de $\mathrm{Ca}^{2+}$ e $\mathrm{Al}(\mathrm{OH})_{4}^{-}$são retirados da solução, proporcionando a retomada da dissolução das partículas de cimento, o que possibilita um contínuo crescimento dos hidratos [5].

Além dos aditivos dispersantes, outros aditivos como $\mathrm{Li}_{2} \mathrm{CO}_{3}$, bem como a presença de matriz, exercem influência na cinética de hidratação do cimento [6-10]. Assim, o principal objetivo deste trabalho foi avaliar a influência dessas variáveis no processo de hidratação de um cimento aluminoso (CA14M) por meio de medidas de temperatura e ensaios reológicos oscilatórios em função do tempo. Também foi avaliada a influência da combinação de aditivos visando a determinação de sistemas de aditivação que venham a favorecer uma boa trabalhabilidade e um curto tempo para a desmoldagem.

\section{MATERIAIS E MÉTODOS}

Os materiais utilizados foram o cimento de aluminato de cálcio CA14M (Almatis-US) e as aluminas, Esy Pump 1000, alumina calcinada A17-NE e A-1000SG (AlmatisUS), cujas características são apresentadas na Tabela I. Tais

Tabela I - Composições químicas e propriedades físicas dos materiais utilizados. [Table I - Chemical compositions and physical properties of the materials used.]

\begin{tabular}{ccccc}
\hline Fases $(\%)$ & CA14M & Esy Pump 1000 & A17-NE & A-1000 $\mathrm{SG}^{*}$ \\
\hline $\mathrm{Al}_{2} \mathrm{O}_{3}$ & 72 & 99,4 & 99,8 & 99,8 \\
$\mathrm{CaO}$ & 27 & - & 0,02 & 0,02 \\
$\mathrm{SiO}_{2}$ & 0,3 & 0,10 & 0,03 & 0,03 \\
$\mathrm{Na}_{2} \mathrm{O}$ & 0,3 & 0,30 & 0,10 & 0,07 \\
Densidade $\left(\mathrm{g} / \mathrm{cm}^{3}\right)$ & 2,96 & 3,99 & 3,94 & 3,95 \\
$\mathrm{ASE} \mathrm{BET}\left(\mathrm{m}^{2} / \mathrm{g}\right)$ & 1,87 & 3,3 & 2,9 & 8,4 \\
$\mathrm{D}_{50}(\mu \mathrm{m})$ & 9,4 & 2,6 & 4,0 & 0,57 \\
Viscosidade $(\mathrm{mPa} . \mathrm{s}), 50 \mathrm{~s}^{-1}$ & $386^{* * *}$ & $4270^{* * * *}$ & $852^{* * * *}$ & $7980^{* *}$ \\
\hline
\end{tabular}

\footnotetext{
** * A matriz A17-NE:A-1000SG (93:7) apresenta ASE = 3,28 $\mathrm{m}^{2} / \mathrm{g}$ e teor de $\mathrm{Na}_{2} \mathrm{O}$ de 0,098\%.

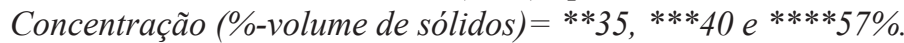


aluminas normalmente constituem as matrizes de concretos refratários sendo utilizadas isoladamente (Esy Pump ou A17-NE) ou combinadas A17-NE:A1000SG (93:7\%). Como aditivo inorgânico foi selecionado o carbonato de

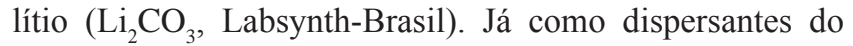
cimento e/ou matriz foram avaliados diferentes compostos: polimetacrilato de sódio (Darvan-7S, Vanderbilt, $2500 \mathrm{~g} /$ mol), ácido cítrico anidro (Labsynth, $192 \mathrm{~g} / \mathrm{mol}$ ), citrato de diamônio (Sigma Aldrich), hexametafosfato de sódio (Labsynth) e os polímeros da família do poliglicol, fornecidos pela SKW Polymers (Castament FS 20, FS 30 e FS 40).

Suspensões aquosas dos materiais estudados foram preparadas para avaliação quanto à eficiência dos aditivos na dispersão. Estas suspensões continham teores de sólidos distintos dado as diferentes distribuições granulométricas e estado de aglomeração das partículas: 40\%-volume (CA14M), 35\%-volume (A-1000SG) ou 57\%-volume (A17NE ou Esy Pump). Esses ensaios consistiram na medida da viscosidade das suspensões em função do teor de dispersante para uma taxa de cisalhamento de $50 \mathrm{~s}^{-1}$, utilizando-se o equipamento Rheostress 300 da ThermoHaake.

A fim de avaliar a influência dos aditivos dispersantes no processo de hidratação, foram preparadas suspensões aquosas de cimento na ausência $(16,8 \%$-v. ou $37 \%$-p de sólidos) e na presença de 50\%-p das aluminas (26\%-v. de sólidos) contendo os aditivos dispersantes $(0,1$ ou $0,9 \mathrm{mg} /$ $\mathrm{m}^{2}$ de pó). Para verificar a influência da combinação de aditivos as suspensões de cimento e cimento-matriz também foram preparadas na presença de $0,1 \mathrm{mg} / \mathrm{m}^{2}(0,05 \%$-p) de dispersante e $\mathrm{Li}_{2} \mathrm{CO}_{3}(0,01 \%$-p sobre a massa de cimento). $\mathrm{O}$ efeito isolado do aditivo inorgânico também foi avaliado utilizando-se suspensões de cimento e cimento-matriz contendo ou não 0,01\%-p de $\mathrm{Li}_{2} \mathrm{CO}_{3}$.

Imediatamente após a mistura com auxílio de um agitador magnético, as suspensões foram submetidas a medidas de temperatura e à ensaios reológicos oscilatórios.

As medidas de temperatura foram monitoradas em função do tempo, com o auxílio de um termopar conectado a um sistema automático de aquisição de dados (ESA 9800, Matec Applied Sciences, UK). Esse sensor foi inserido nas suspensões de cimento ou cimento-matriz sendo as medidas imediatamente efetuadas. O sistema para o controle de temperatura foi especialmente desenvolvido para permitir medidas na temperatura desejada $\left(50^{\circ} \mathrm{C}\right)$.

Os ensaios reológicos oscilatórios em função do tempo foram realizados utilizando-se o equipamento Rheostress 300 da ThermoHaake. As medidas reológicas obtidas nesses ensaios são o módulo de armazenamento (G'), que representa às características elásticas da suspensão, e o módulo de perda G”, que está associado às propriedades viscosas do material. Tais medidas devem ser realizadas na região onde o material apresenta um regime viscoelástico linear, ou seja, para uma combinação de tensão $(\tau)$ e freqüência (f), onde G' permaneça constante, preservando assim a estrutura do material em curtos intervalos de tempo. Dessa forma, testes preliminares com variação de $\tau$ e $\mathrm{f}$ foram realizados para cada tipo de suspensão a fim de determinar os valores a serem adotados em cada caso. Tais ensaios também foram executados com controle de temperatura em $50{ }^{\circ} \mathrm{C}$.

Adicionalmente, medidas de condutividade elétrica em função do tempo foram realizadas para o cimento CA14M com controle de temperatura em $50{ }^{\circ} \mathrm{C}$, utilizando-se um eletrodo de condutividade (ESA 9800, Matec Applied Sciences - UK). Suspensões aquosas de cimento foram preparadas com um teor de sólidos de 4,2\% em volume para a realização das medidas na ausência ou na presença de dispersante $\left(0,05 \%\right.$-p) e $\mathrm{Li}_{2} \mathrm{CO}_{3}(0,01 \%$-p) quando puros ou combinados. Suspensões aquosas das diferentes matrizes foram preparadas com um teor de sólidos de 16,8\% em volume para medidas de condutividade a $50^{\circ} \mathrm{C}$.

\section{RESULTADOS E DISCUSSÃO}

Geralmente, na hidratação de um cimento de aluminato de cálcio se verifica, inicialmente, uma pequena geração de calor. Segue um período dormente após o qual a precipitação de hidratos ocorre e um correspondente aumento na evolução de calor é observado [11]. Dessa forma, por meio da introdução de um termopar na pasta de cimento o aumento de temperatura pode ser detectado e usado como medida das suas reações de hidratação e conseqüente endurecimento.

As medidas do módulo de armazenamento permitem verificar a influência do fenômeno de hidratação nas interações coloidais e no comportamento de pega inicial [12]. Com a consolidação da suspensão é verificado um aumento de G' e uma dispersão dos valores de G", a medida que o módulo de armazenamento se aproxima de um valor constante (aproximadamente $10^{6} \mathrm{~Pa}$ ).

Neste trabalho, medidas de temperatura e ensaios reológicos oscilatórios foram realizados para suspensões de cimento puro ou quando preparadas na presença de matriz. Exemplos são apresentados na Fig. 1 para as suspensões de CA14M na presença de finos (Esy Pump ou A17NE: A1000SG). Nesta figura são indicadas as tangentes das curvas de temperatura e G', cujas intersecções foram tomadas como o tempo de pega ou endurecimento do cimento.

Entretanto, ao contrário do verificado em suspensões de cimento puro [13], os tempos de pega determinados por meio das medidas de temperatura e ensaios reológicos para as suspensões cimento-matriz não foram coincidentes (Fig. 1).

Tal comportamento pode ser explicado visto que a precipitação de hidratos, bem como, a consolidação da suspensão é influenciada pela presença de matriz. As partículas da matriz constituem sítios de nucleação a partir dos quais ocorrerá o crescimento dos hidratos. Comparandose as viscosidades obtidas para as suspensões de Esy Pump e A17-NE na ausência de dispersante (Tabela I), o superior valor obtido no caso da Esy Pump indica um estado de maior aproximação entre as suas partículas. Dessa forma, tão logo os cristais de hidratos comecem a precipitar (início 

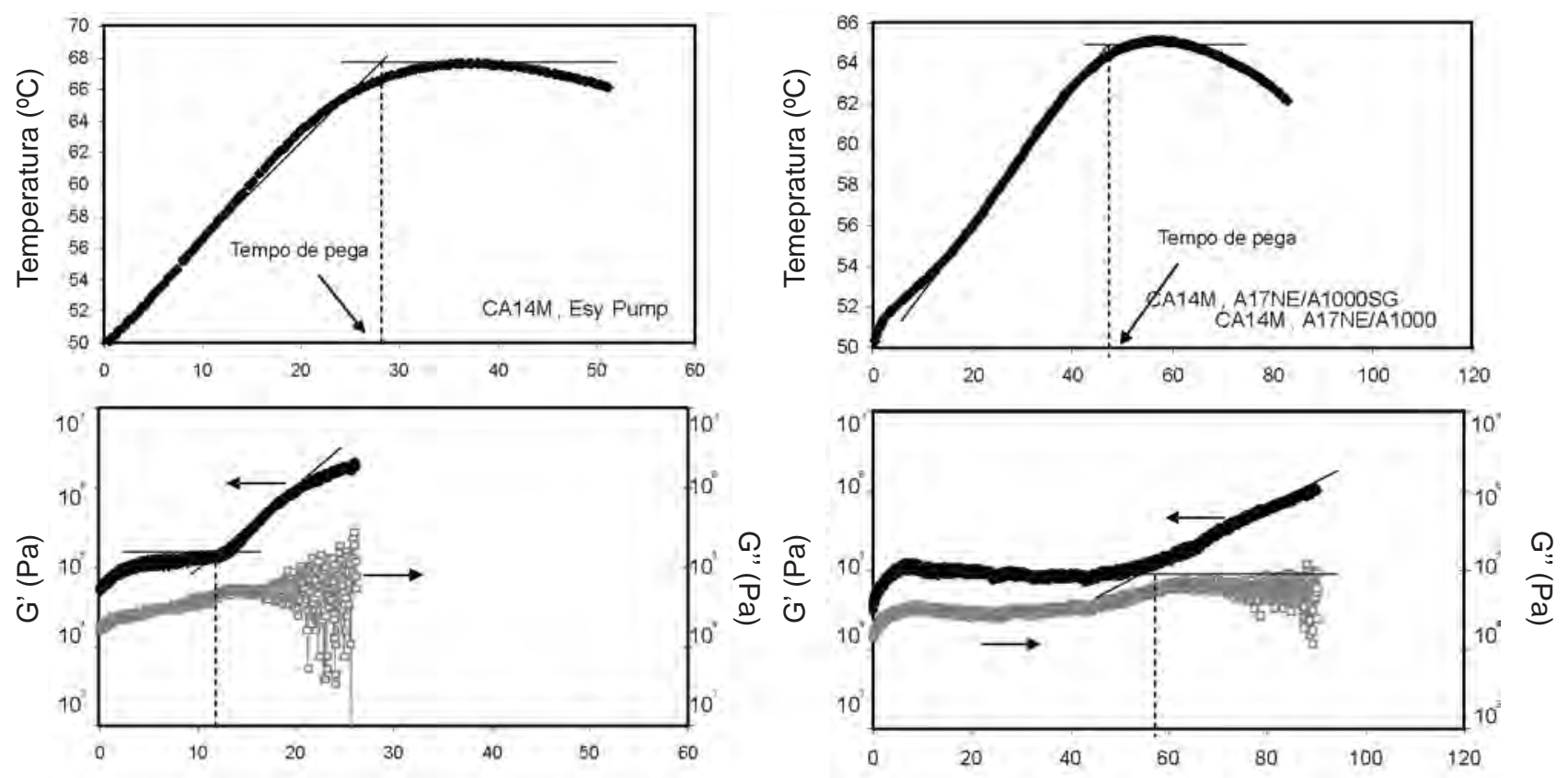

Tempo (min)

Figura 1: Medidas de temperatura e ensaios reológicos oscilatórios, em função do tempo, para suspensões aquosas de cimento CA14M na presença de finos (50\%-p do ligante) mantendo-se a temperatura do banho térmico em $50{ }^{\circ} \mathrm{C}$.

[Figure 1: Temperature measurements and oscillatory rheological tests as a function of the time for aqueous suspensions of cement (CA14M) with fines $\left(50 w t \%\right.$ of binder) at $\left.50^{\circ} \mathrm{C}.\right]$

do aumento de temperatura) e crescer na superfície das partículas de alumina, ocorrerá o entrelaçamento destes cristais devido a reduzida distância entre as partículas, resultando no aumento de G' (consolidação da suspensão). Isso pode explicar porque é verificado um tempo de pega tão rápido quando medido por meio dos ensaios reológicos. Por outro lado, o entrelaçamento dos cristais torna-se mais difícil quando estes crescem a partir de partículas mais afastadas (menor viscosidade), verificando-se um atraso do aumento de G'.

Dessa forma, para a avaliação quanto à influência das variáveis (matriz, aditivo inorgânico e dispersante) sobre o processo de hidratação do cimento CA14M, foram considerados apenas os tempos de pega obtidos por meio das medidas de temperatura. Estudos complementares estão sendo realizados visando correlacionar os resultados obtidos por meio dos ensaios oscilatórios com o tempo de desmoldagem.

\section{Avaliação quanto à influência da matriz e aditivo inorgânico}

O período de indução (tempo de espera para que se atinjam condições para precipitação) é encurtado com a adição de partículas à suspensão de cimento uma vez que essas constituem sítios de nucleação que eliminam a barreira de energia necessária para nucleação do hidrato [1].

Este encurtamento do período de indução e conseqüente decréscimo do tempo de pega são observados para a mistura de cimento com as diferentes matrizes, como apresentado na Fig. 2. Esta redução no tempo depende da área superficial específica (ASE) da matriz presente na pasta de cimento. Quanto maior a ASE da alumina mais pronunciado é o encurtamento do período de indução durante o processo de hidratação [6]. Entretanto, as aluminas usadas apresentam ASE similares, mas, diferentes teores de $\mathrm{Na}_{2} \mathrm{O}$ (Tabela I). Aluminas com maior teor de $\mathrm{Na}_{2} \mathrm{O}$ resultam nos mais curtos períodos de indução durante a hidratação da sua mistura com cimento $[1,6]$. De fato, a alumina Esy Pump possui o maior teor deste óxido, resultando nos menores valores de tempo de pega. A presença de um maior teor de $\mathrm{Na}^{+}$em solução, quando utilizada a Esy Pump, foi confirmada pelo significativo aumento de condutividade quando comparado as demais matrizes, como pode ser visto na Fig. 3 .

A redução no tempo de pega causada pela presença de íons sódio, provenientes dos finos, é resultado da formação de $\mathrm{NaAl}(\mathrm{OH})_{4}$. Com a formação de tal composto ocorre um desequilíbrio entre as concentrações dos íons $\mathrm{Ca}^{2+} \mathrm{e} \mathrm{Al}(\mathrm{OH})_{4}^{-}$, resultantes da dissolução do cimento.

É verificado [14] que a relação entre as concentrações desses íons em solução determina qual hidrato será formado em uma determinada temperatura. Para a temperatura de $25{ }^{\circ} \mathrm{C}$ e relação entre $\left[\mathrm{Ca}^{2+}\right]$ e $\left[\mathrm{Al}(\mathrm{OH})_{4}^{-}\right]$próximas a 1 , a formação do hidrato $\mathrm{CAH}_{10}$ é favorecida. Com o aumento dessa relação, ou seja, para maiores teores de $\mathrm{Ca}^{2+}$, passase a favorecer a formação de $\mathrm{C}_{2} \mathrm{AH}_{8}$. Já para maiores 


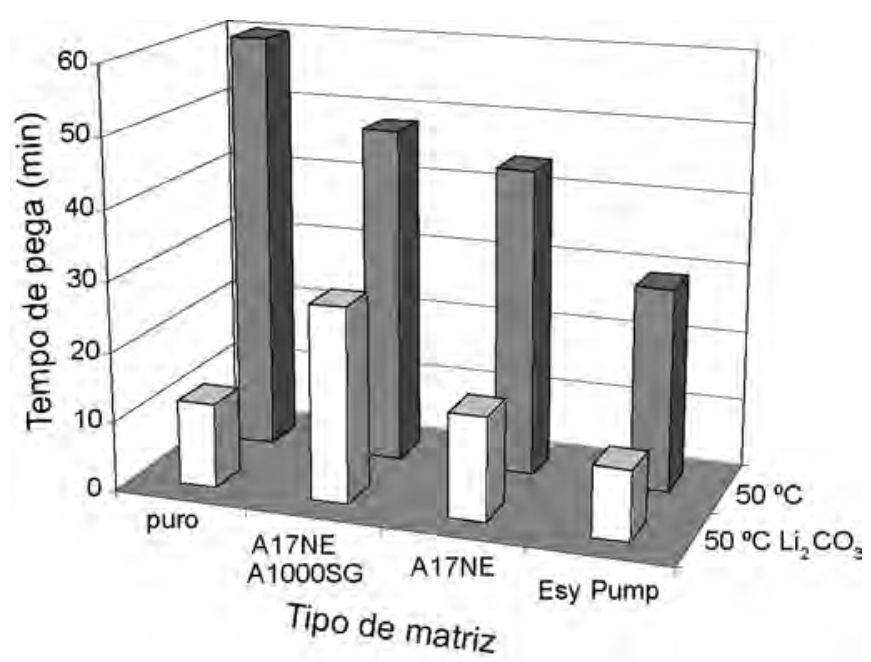

Figura 2: Tempos de pega avaliados por meio de medidas de temperatura em função do tempo, para suspensões aquosas de cimento $\mathrm{CA} 14 \mathrm{M}$ e cimento-matriz, contendo ou não $\mathrm{Li}_{2} \mathrm{CO}_{3}$ (0,01\%p), para a temperatura de ensaio de $50{ }^{\circ} \mathrm{C}$.

[Figure 2: Setting time evaluated through temperature measurements as a function of the time for aqueous suspensions of the CA14M cement and cement-matrix with or without $\mathrm{Li}_{2} \mathrm{CO}_{3}$ (0.01wt\%), at $50^{\circ} \mathrm{C}$.]

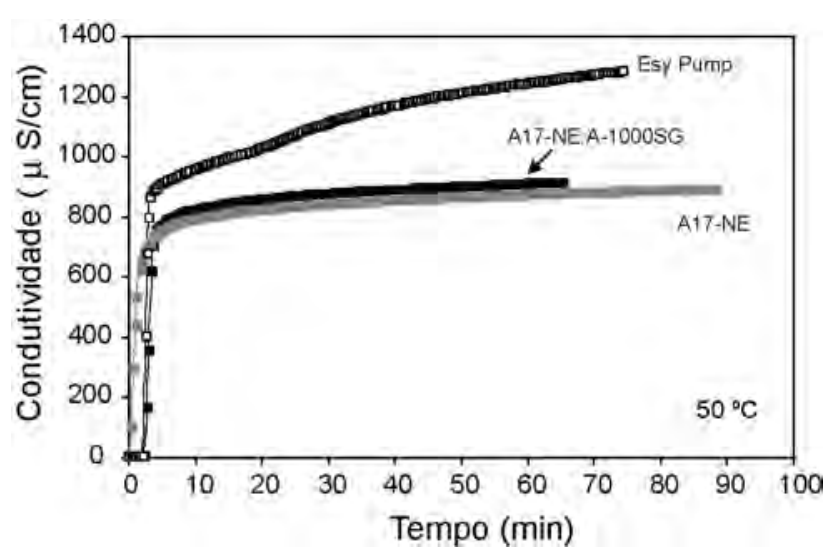

Figura 3: Curvas de condutividade elétrica em função do tempo obtidas para suspensões aquosas das diferentes matrizes para a temperatura de $50{ }^{\circ} \mathrm{C}$.

[Figure 3: Electrical conductivity as a function of the time obtained for aqueous suspensions of the different aluminas, at $50{ }^{\circ} \mathrm{C}$.]

concentrações de $\mathrm{Al}(\mathrm{OH})_{4}^{-}$é predominante a formação de $\mathrm{AH}_{3}$. Entretanto, para a temperatura de ensaio utilizada neste trabalho, $50{ }^{\circ} \mathrm{C}$, o hidrato majoritariamente formado é o $\mathrm{C}_{3} \mathrm{AH}_{6}$.

Esses tipos de hidratos formados diferem quanto ao grau de solubilidade, na seguinte ordem $\mathrm{C}_{3} \mathrm{AH}_{6}<\mathrm{C}_{2} \mathrm{AH}_{8}<\mathrm{CAH}_{10}$ $<\mathrm{AH}_{3}$. A formação do tipo menos solúvel implica numa alta velocidade de precipitação reduzindo o tempo de pega do cimento. Um raciocínio semelhante pode ser aplicado para se entender o atraso no tempo de pega quando o hidrato favorecido é o $\mathrm{AH}_{3}$, que inicialmente tem uma solubilidade maior que $\mathrm{CAH}_{10}$ e por esse motivo requer um maior período de indução.

Deste modo, por meio da formação do composto $\mathrm{NaAl}(\mathrm{OH})_{4}$, é favorecida a precipitação do hidrato menos solúvel, principalmente quando na presença da matriz mais rica em sódio (Esy Pump).

Os tempos de precipitação de hidratos também foram avaliados para suspensões de cimento e cimento-matriz na presença do aditivo inorgânico $\mathrm{Li}_{2} \mathrm{CO}_{3}$, para a temperatura de $50{ }^{\circ} \mathrm{C}$ (Fig. 2). Observa-se uma considerável redução no tempo de pega na presença deste aditivo uma vez que os íons $\mathrm{Li}^{+}$proporcionam a formação de compostos insolúveis do tipo $\mathrm{LiAl}(\mathrm{OH})_{4}$, retirando íons $\mathrm{Al}(\mathrm{OH})_{4}^{-}$da solução $[9,10]$. Dessa forma, os sais de lítio também atuam na reação de hidratação do cimento favorecendo a formação de hidratos menos solúveis que irão precipitar mais facilmente.

Além disso, a precipitação desses compostos insolúveis de lítio é mais rápida do que a precipitação dos hidratos de aluminato de cálcio e apresentam estrutura cristalina necessária para servir de germes para a nucleação do hidrato $\mathrm{C}_{2} \mathrm{AH}_{8}$, independente da temperatura. Isso faz com que a barreira de energia necessária à nucleação desse hidrato deixe de existir.

Quando comparado o efeito da adição de $\mathrm{Li}_{2} \mathrm{CO}_{3}$ sobre o cimento puro, com a adição na suspensão de cimento combinado com a matriz, observa-se que seu efeito é reduzido na presença de matriz. Esse comportamento pode ser entendido considerando-se que, quando se adiciona $\mathrm{Li}^{+}$no sistema cimento/matriz o aumento na razão entre $\left[\mathrm{Ca}^{2+}\right]$ e $\left[\mathrm{Al}(\mathrm{OH})_{4}^{-}\right]$é menor que aquele observado para o caso do cimento puro (considerando os íons $\mathrm{Al}(\mathrm{OH})_{4}$ provenientes da alumina). Além disso, nessa situação, verifica-se a presença de sítios de nucleação tanto para o $\mathrm{C}_{2} \mathrm{AH}_{8}$ (formados pela precipitação do $\mathrm{LiAl}(\mathrm{OH})_{4}^{-}$) quanto para o $\mathrm{AH}_{3}$ (devido a presença da matriz). Entretanto, como a razão entre as concentrações dos íons provenientes da dissolução do cimento não aumenta como no caso da adição de $\mathrm{Li}^{+}$na pasta de cimento puro, a formação do $\mathrm{AH}_{3}$ deve prevalecer. Dessa forma, a menor redução observada no tempo de pega causada pela adição de $\mathrm{Li}_{2} \mathrm{CO}_{3}$ na suspensão cimento/matriz pode ser atribuída a não predominância na formação do hidrato menos solúvel $\left(\mathrm{C}_{2} \mathrm{AH}_{8}\right)$.

Avaliação quanto à influência dos dispersantes e da combinação dispersante- $\mathrm{Li}_{2} \mathrm{CO}_{3}$

Inicialmente foram realizadas medidas de viscosidade das suspensões de cimento e das diferentes matrizes a fim de se determinar o comportamento dos aditivos dispersantes. Os resultados do teor necessário para promover a menor viscosidade da suspensão em cada caso são apresentados na Tabela II. 
Tabela II - Viscosidades mínimas obtidas para suspensões de CA14M (40\%-volume), Esy Pump ou A17-NE (57\%-volume) e A-1000SG (35\%-volume) na presença de um teor ótimo de aditivo.

[Table II - Viscosity measurements of suspensions containing, CA14M (40 vol\%), Esy Pump or A17-NE (57 vol\%) and A1000SG (35 vol\%) prepared with optimum additive content.]

\begin{tabular}{|c|c|c|c|c|c|}
\hline \multirow[t]{2}{*}{ Aditivos } & \multirow[t]{2}{*}{$\begin{array}{l}\text { Teor ótimo } \\
\left(\mathrm{mg} / \mathrm{m}^{2}\right)\end{array}$} & \multicolumn{4}{|c|}{$\begin{array}{l}\text { Materiais } \\
\text { viscosidades mínimas (mPa.s), } 50 \mathrm{~s}^{-1}\end{array}$} \\
\hline & & CA14M & Esy pump & A17-NE & A-1000SG \\
\hline $\mathrm{s} /$ aditivo & 0 & 386 & 4270 & 852 & 7980 \\
\hline Darvan-7S & 0,1 & 454 & 264 & 412 & 59 \\
\hline Ácido cítrico & 0,1 & 381 & 93 & 190 & 95 \\
\hline Citrato diamônio & 0,1 & 419 & 93 & 192 & 55 \\
\hline HMF-Na & $\begin{array}{l}0,1 \\
0,2\end{array}$ & 487 & 148 & 190 & 34 \\
\hline FS20 & 0,1 & 456 & 107 & 209 & 56 \\
\hline FS 30 & $\begin{array}{l}0,1 \\
0,2 \\
0,9\end{array}$ & 83 & 2626 & 381 & 130 \\
\hline FS40 & $\begin{array}{l}0,1 \\
0,2 \\
0,3 \\
0,9\end{array}$ & 97 & 162 & 211 & 59 \\
\hline
\end{tabular}

Em seguida, foi avaliada a influência desses aditivos dispersantes no processo de hidratação do cimento CA14M puro ou quando na presença de diferentes aluminas. Estes ensaios permitiram a avaliação quanto à influência da dispersão do cimento e/ou matriz no processo de hidratação desse ligante. Os teores avaliados dos aditivos dispersantes são correspondentes aos teores ótimos observados na Tabela II $\left(0,1\right.$ ou $\left.0,9 \mathrm{mg} / \mathrm{m}^{2}\right)$. A Fig. 4 apresenta os resultados para as suspensões de cimento isentas de matriz. Observa-se que com exceção do hexametafosfato de sódio (HMF-Na), todos os aditivos promoveram o retardamento do tempo de pega, principalmente para o teor de $0,9 \mathrm{mg} / \mathrm{m}^{2}$, quando comparado ao tempo na ausência de aditivo (60 min, Fig. 2).

De uma maneira geral o mecanismo de atuação de aditivos retardadores é atribuído a um aumento no tempo requerido para que o processo de dissolução das fases anidras do cimento proporcione os valores de concentrações de $\mathrm{Ca}^{2+} \mathrm{e} \mathrm{Al}(\mathrm{OH})_{4}^{-}$, necessárias para se iniciar o período de indução (saturação da solução). Neste caso, quanto maior o efeito retardante do aditivo, mais difícil é o processo de dissolução.

Dessa forma, considerando apenas o poder dispersante dos aditivos avaliados, o FS30 e o FS40 deveriam facilitar a dissolução das partículas de cimento devido a eficiência verificada quanto a dispersão dessas partículas (Tabela
II). Por outro lado, os demais aditivos não promoveram a dispersão e deveriam atuar como bons retardadores do tempo

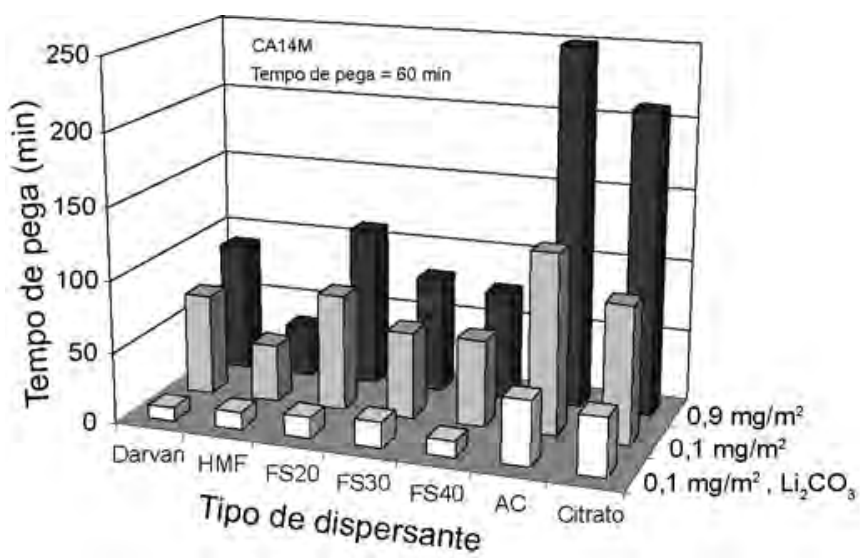

Figura 4: Tempos de pega avaliados por meio de medidas de temperatura em função do tempo, para suspensões aquosas de cimento CA14M na presença de diferentes dispersantes $(0,1$ ou $\left.0,9 \mathrm{mg} / \mathrm{m}^{2}\right)$ ou combinados com $0,01 \%-\mathrm{p} \mathrm{Li}_{2} \mathrm{CO}_{3}\left(0,1 \mathrm{mg} / \mathrm{m}^{2}\right)$, para a temperatura de ensaio de $50{ }^{\circ} \mathrm{C}$.

[Figure 4: Setting time evaluated through temperature measurements as a function of the time for aqueous suspensions of the cement CA14M with different dispersants $\left(0.1\right.$ or $\left.0.9 \mathrm{mg} / \mathrm{m}^{2}\right)$ or with dispersant-0.01wt\% $\mathrm{Li}_{2} \mathrm{CO}_{3}\left(0.1 \mathrm{mg} / \mathrm{m}^{2}\right)$, at $50{ }^{\circ} \mathrm{C}$.] 
de pega. Entretanto, não foi verificado um efeito acelerador quando na presença dos aditivos FS30 e FS40, como também o HMF-Na não apresentou o maior efeito retardador, apesar do seu pior efeito dispersante.

Assim, deve haver outros fatores, além do poder dispersante, que determinam o retardamento do tempo de pega por parte dos aditivos. De fato, existem outras maneiras pelas quais compostos químicos podem proporcionar atraso no tempo de pega do cimento [8-10]. Os ânions produzidos por esses compostos em solução são formados por grupos R-COO- e R-OH que possuem grande afinidade pelo cálcio. A forte interação desses ânions em solução em relação ao $\mathrm{Ca}^{2+}$ tem duas conseqüências no processo de hidratação do cimento. A primeira delas consiste no fato de que a reação estequiométrica entre os ânions e o $\mathrm{Ca}^{2+}$ produz sais insolúveis em $\mathrm{pH}$ alcalino ( $\mathrm{pH}$ do meio contendo cimento) o que causa uma diminuição da relação entre as concentrações dos íons $\mathrm{Ca}^{2+}$ e $\mathrm{Al}(\mathrm{OH})_{4}^{-}$. Assim, a nucleação e crescimento dos hidratos de aluminato de cálcio são mais lentos, já que a fase mais solúvel $\left(\mathrm{AH}_{3}\right)$ é favorecida. A segunda conseqüência está relacionada ao fato de que a forte interação dos ânions gerados em solução com o cálcio possibilita a formação de sais insolúveis mesmo na interface sólido-líquido formada pela partícula do cimento e a solução. Nesse caso, a formação desses sais proporciona o surgimento de uma camada sobre a superfície das partículas de cimento. A formação dessa camada dificulta o prosseguimento da reação do cimento com a água, atrasando o estágio de saturação da solução.

No caso de todos os sistemas cimento-matriz os aditivos também promoveram o retardamento do tempo de pega (Figs. 5-7), com exceção do HMF-Na, quando comparado aos tempos na ausência de dispersante (Fig. 2).

Entretanto, o efeito retardador promovido pelos aditivos ácido cítrico e citrato são consideravelmente superiores. Esse comportamento deve-se principalmente ao fato de que os aditivos apresentam número de sítios ionizáveis e constantes ácidas distintas, sendo que o $\mathrm{AC}$ e o citrato devem apresentar mais sítios ionizáveis e também as maiores constantes ácidas, produzindo assim maior número de ânions com grande afinidade pelo cálcio.

Esses resultados também mostraram que a influência de aditivos dispersantes na dissolução de íons e formação de hidratos é predominante na determinação do tempo de pega comparativamente a etapa de precipitação de hidratos. Justamente os aditivos (AC e citrato) que se apresentam como os aditivos mais eficientes na dispersão das matrizes estudadas (Tabela II) apresentaram o maior poder retardador. A superior dispersão das partículas da matriz deveria resultar em mais sítios nucleantes facilitando a precipitação de hidratos e portanto deveria resultar no menor efeito retardador.

Além disso, independente do aditivo e teor avaliado o tempo de pega diminuiu com a mistura da matriz ao cimento na seguinte ordem A17-A1000, A17NE e Esy pump. Isso concorda com os resultados apresentados anteriormente, ou seja, a redução no tempo de indução foi mais pronunciado à medida que o teor de $\mathrm{Na}_{2} \mathrm{O}$ do sistema aumentou.

Cabe ressaltar que o ácido cítrico apresentou um superior efeito retardador quando comparado ao citrato de diamônio, independente do sistema estudado. O processo de nucleação de hidratos envolve a ligação entre grupos $\mathrm{OH}$ de

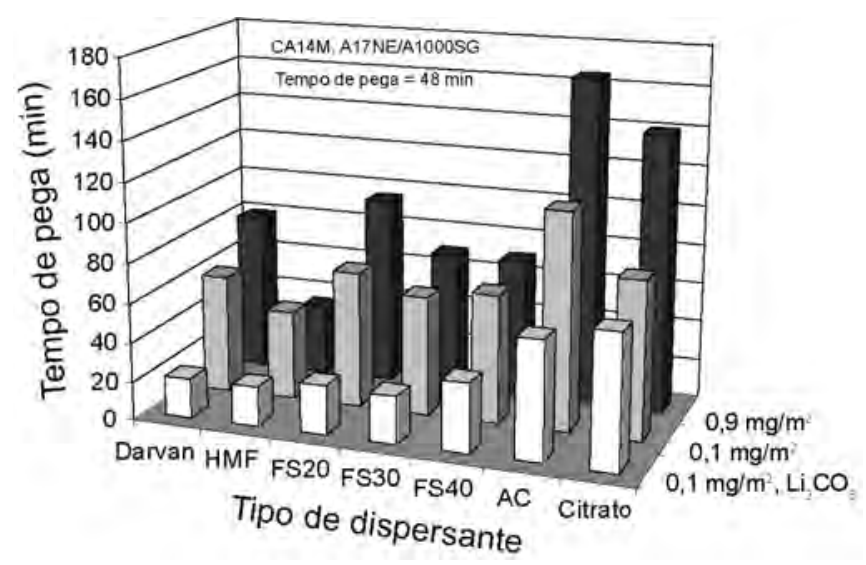

Figura 5: Tempos de pega avaliados por meio de medidas de temperatura em função do tempo, para suspensões aquosas de cimento-A17NE/A1000SG (50:50\%-p) na presença de diferentes dispersantes $\left(0,1\right.$ ou $\left.0,9 \mathrm{mg} / \mathrm{m}^{2}\right)$ ou combinados com $0,01 \%-\mathrm{p} \mathrm{Li}_{2} \mathrm{CO}_{3}$ $\left(0,1 \mathrm{mg} / \mathrm{m}^{2}\right)$, para a temperatura de ensaio de $50{ }^{\circ} \mathrm{C}$.

[Figure 5: Setting time evaluated through temperature measurements as a function of the time for aqueous suspensions of the cement-A17NE/ A1000SG (50:50 wt\%) with different dispersants $\left(0.1 \mathrm{or} 0.9 \mathrm{mg} / \mathrm{m}^{2}\right)$ or with dispersant- $0.01 \mathrm{wt} \% \mathrm{Li}_{2} \mathrm{CO}_{3}\left(0.1 \mathrm{mg} / \mathrm{m}^{2}\right)$, at $50{ }^{\circ} \mathrm{C}$.]

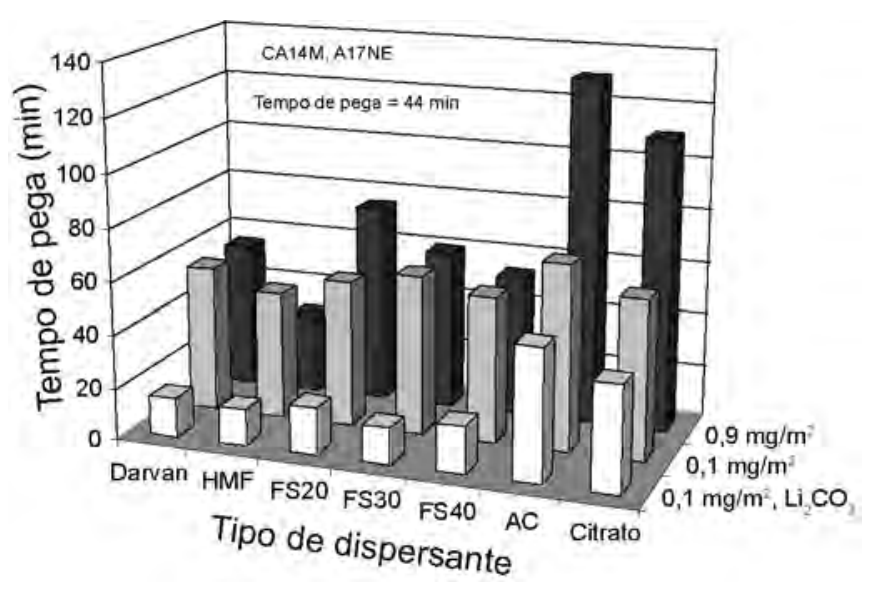

Figura 6: Tempos de pega avaliados por meio de medidas de temperatura em função do tempo, para suspensões aquosas de cimento-A17NE (50:50\%-p) na presença de diferentes dispersantes $\left(0,1 \mathrm{ou} 0,9 \mathrm{mg} / \mathrm{m}^{2}\right)$ ou combinados com $0,01 \%-\mathrm{p} \mathrm{Li}_{2} \mathrm{CO}_{3}\left(0,1 \mathrm{mg} / \mathrm{m}^{2}\right)$, para a temperatura de ensaio de $50{ }^{\circ} \mathrm{C}$.

[Figure 6: Setting time evaluated through temperature measurements as a function of the time for aqueous suspensions of the cementA17NE (50:50 wt\%) with different dispersants $\left(0.1 \mathrm{or} 0.9 \mathrm{mg} / \mathrm{m}^{2}\right)$ or with dispersant- $0.01 \mathrm{wt} \% \mathrm{Li}_{2} \mathrm{CO}_{3}\left(0.1 \mathrm{mg} / \mathrm{m}^{2}\right)$, at $50^{\circ} \mathrm{C}$.] 


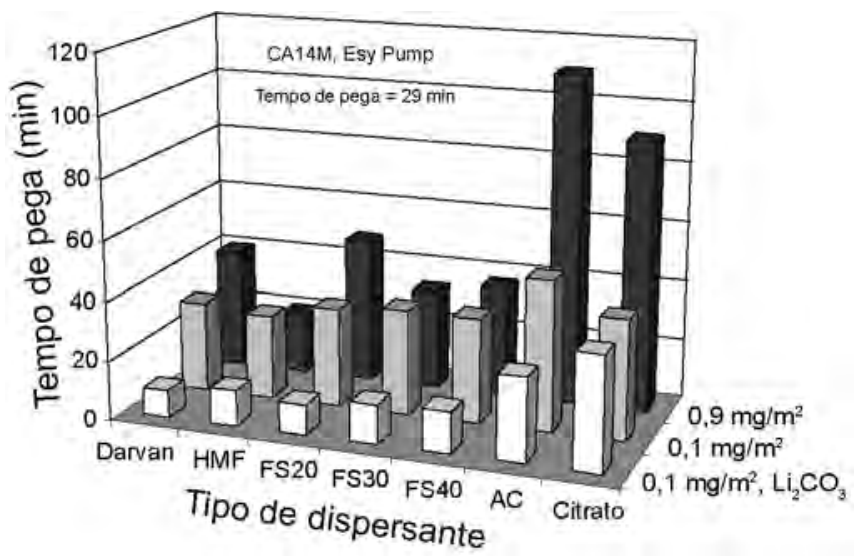

Figura 7: Tempos de pega avaliados por meio de medidas de temperatura em função do tempo, para suspensões aquosas de cimento-Esy Pump (50:50\%-p) na presença de diferentes dispersantes $\left(0,1\right.$ ou $\left.0,9 \mathrm{mg} / \mathrm{m}^{2}\right)$ ou combinados com $0,01 \%$-p $\mathrm{Li}_{2} \mathrm{CO}_{3}\left(0,1 \mathrm{mg} / \mathrm{m}^{2}\right)$, para a temperatura de ensaio de $50{ }^{\circ} \mathrm{C}$.

[Figure 7: Setting time evaluated throughtemperature measurements as a function of the time for aqueous suspensions of the cement-Esy Pump (50:50 wt\%) with different dispersants $\left(0.1\right.$ or $\left.0.9 \mathrm{mg} / \mathrm{m}^{2}\right)$ or with dispersant-0.01 $\mathrm{wt} \% \mathrm{Li}_{2} \mathrm{CO}_{3}\left(0.1 \mathrm{mg} / \mathrm{m}^{2}\right)$, at $50{ }^{\circ} \mathrm{C}$.]

uma molécula de aluminato de cálcio hidratado com outra molécula desse composto, através da sobreposição do par de elétrons isolado do oxigênio com um orbital vazio do átomo de cálcio [9]. Íons hidrogênio podem se ligar fortemente aos grupos $\mathrm{OH}$ dos hidratos bloqueando o processo de nucleação e conseqüentemente retardando a precipitação de hidratos. Por esse motivo, o fato do ácido cítrico apresentar mais hidrogênios ionizáveis pode explicar seu superior efeito retardador.

Quanto à combinação dos aditivos dispersantes com o acelerador $\mathrm{Li}_{2} \mathrm{CO}_{3}$, as Figs. 4-7 mostram que o efeito do acelerador não foi modificado pela presença dos aditivos dispersantes, como exceção para os aditivos AC e citrato. Observa-se que nestes casos ocorreu uma combinação dos efeitos retardador (AC e citrato) e acelerador $\left(\mathrm{Li}_{2} \mathrm{CO}_{3}\right)$, obtendo-se um tempo de pega intermediário.

$\mathrm{O}$ efeito observado quanto à influência dos diferentes aditivos dispersantes, bem como da combinação entre eles, foi confirmada por meio de medidas de condutividade realizadas para as suspensões aquosas de CA14M puro e na presença de dispersante $\left(0,05 \%\right.$-p) e $\mathrm{Li}_{2} \mathrm{CO}_{3}(0,01 \%$-p) quando puros ou combinados para a temperatura de $50^{\circ} \mathrm{C}$ (Figs. 8 e 9). A dissolução dos íons $\mathrm{Ca}^{2+} \mathrm{e} \mathrm{Al}(\mathrm{OH})_{4}^{-}$das partículas de cimento em água causa um aumento da condutividade elétrica das suspensões aquosas de cimento no estágio inicial do processo de hidratação. Posteriormente, verifica-se uma queda de condutividade relacionada a precipitação de hidratos [15]. Cabe ressaltar que os tempos de pega observados diferem dos medidos por meio dos ensaios de temperatura, o que pode ser atribuído as diferentes metodologias adotadas para a execução destes ensaios.
Esses resultados confirmam que os aditivos $\mathrm{AC}$ e citrato atuam aumentando o tempo de indução (patamar de condutividade, Fig. 8) pelo fato dos ânions produzidos por esses compostos seqüestrarem os íons cálcio. Dessa forma, quando esses aditivos são utilizados em conjunto com o acelerador, o efeito deste último como facilitador da precipitação de hidratos fica prejudicado. Isso mostra que não basta existir germes que facilitem a precipitação de um determinado hidrato, mas deve também haver uma concentração de íons em solução suficientes para a sua formação. Na presença de AC é observado um tempo de indução maior (Fig. 8) confirmando seu superior efeito retardador pela dificuldade imposta à formação dos hidratos. Este comportamento está associado à maior quantidade de hidrogênios ionizáveis, quando comparado ao citrato, como apresentado na Fig. 10.

Uma vez que a ação dos aditivos retardadores e aceleradores se dão em estágios diferentes no processo de hidratação [16] uma combinação de aditivos pode ser planejada de acordo com o tempo de trabalhabilidade necessário reduzindo o tempo para a desmoldagem do corpo conformado.

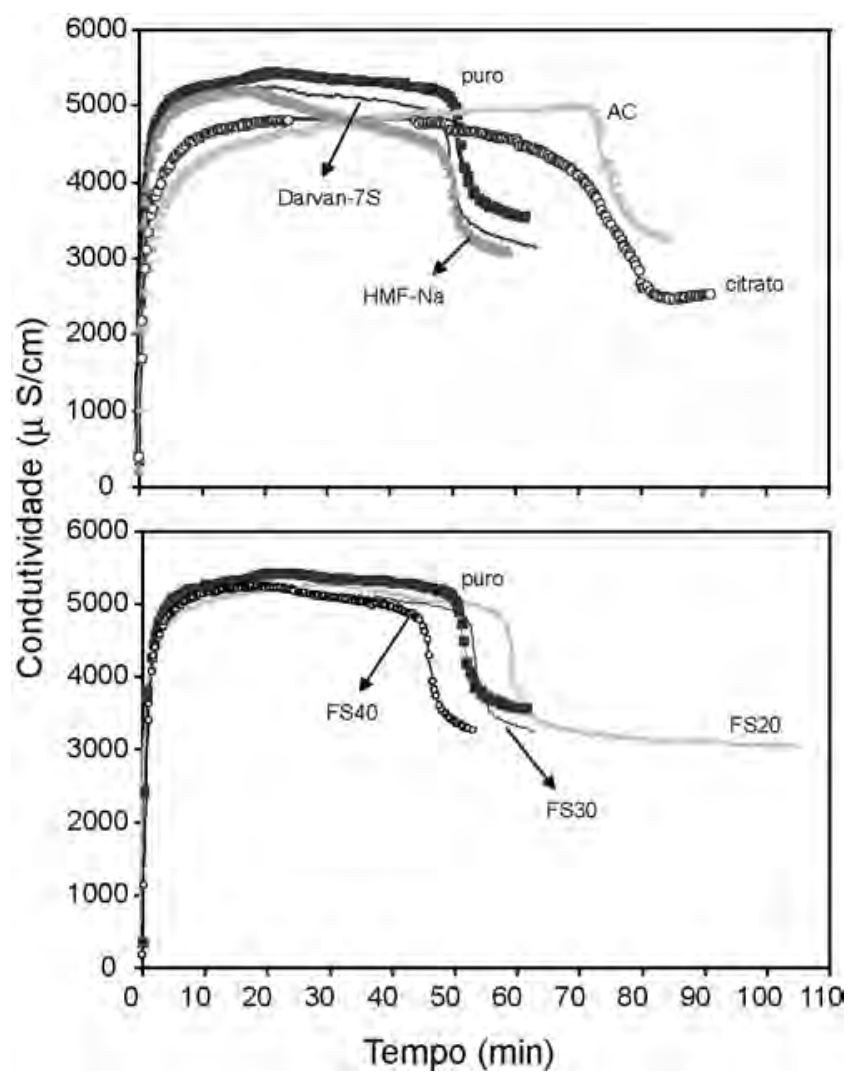

Figura 8: Curvas de condutividade elétrica em função do tempo, obtidas para suspensões aquosas de CA14M puro e na presença de diferentes dispersantes $\left(0,05 \%\right.$-p) para a temperatura de $50{ }^{\circ} \mathrm{C}$.

[Figure 8: Electrical conductivity as a function of the time obtained for aqueous suspensions of the pure cement CA14M and with different dispersants (0.05 wt.\%), at $50{ }^{\circ} \mathrm{C}$.] 


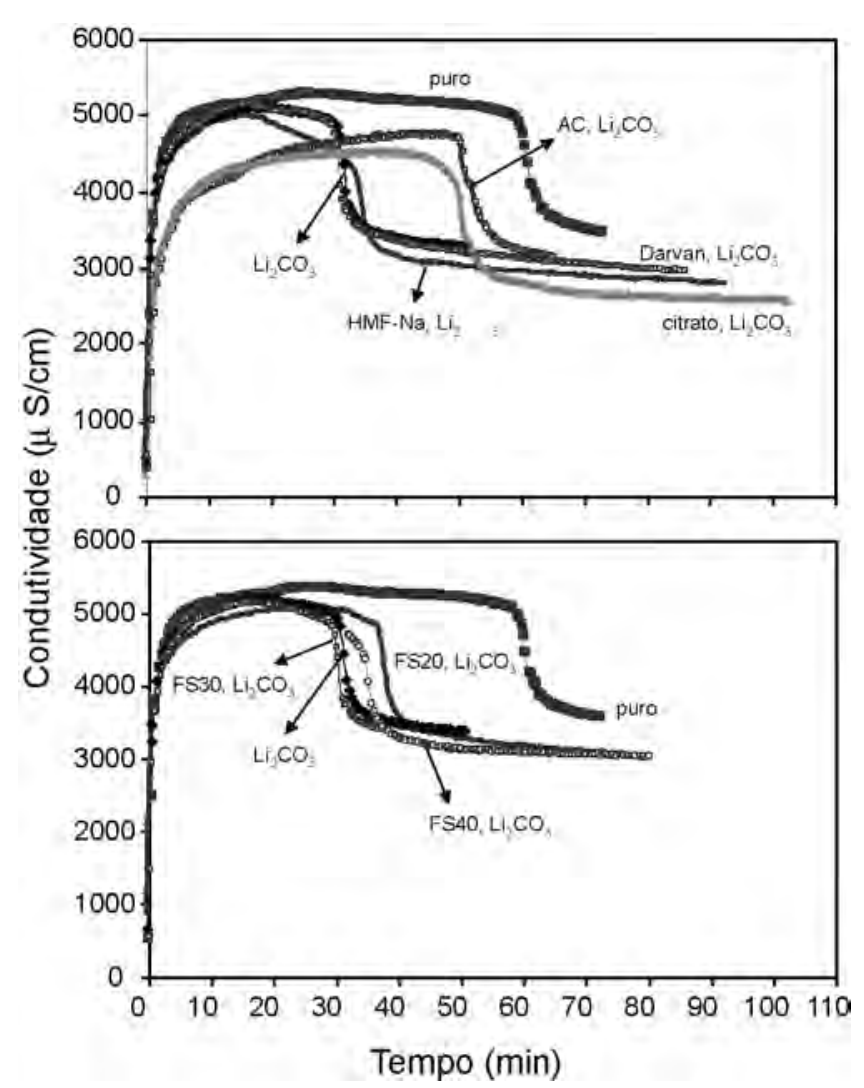

Figura 9: Curvas de condutividade elétrica em função do tempo obtidas para suspensões aquosas de CA14M puro e na presença de $\mathrm{Li}_{2} \mathrm{CO}_{3}\left(0,01 \%\right.$-p) ou $\mathrm{Li}_{2} \mathrm{CO}_{3}$-dispersante $(0,05 \%$-p) para a temperatura de $50{ }^{\circ} \mathrm{C}$.

[Figure 9: Electrical conductivity as a function of the time obtained for aqueous suspensions of the pure cement CA14M and with $\mathrm{Li}_{2} \mathrm{CO}_{3}(0.01 \mathrm{wt} \%)$ or $\mathrm{Li}_{2} \mathrm{CO}_{3}$-dispersant $(0.05 \mathrm{wt} \%)$, at $50^{\circ} \mathrm{C}$.]

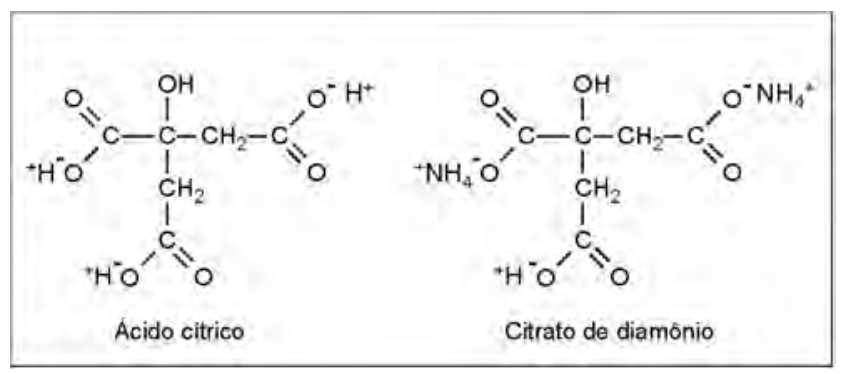

Figura 10: Estrutura química dos principais aditivos retardadores do processo de hidratação do cimento de aluminato de cálcio. [Figure 10: Chemical structures of main retarders additives of hydration process of calcium aluminate cement.]

\section{CONCLUSÃo}

Os tempos de pega determinados por meio dos ensaios reológicos e medidas de temperatura para as suspensões cimento-matriz não são coincidentes devido ao estado de dispersão das partículas da matriz determinar a velocidade de consolidação da suspensão.
A presença de matriz também promove redução do tempo de pega, devido as suas partículas constituírem sítios de nucleação facilitando a precipitação de hidratos. No entanto, tal redução do tempo de pega pode ser principalmente atribuída ao teor de $\mathrm{Na}_{2} \mathrm{O}$ das diferentes matrizes. $\mathrm{O}$ aumento do sódio liberado em solução favorece a precipitação do hidrato menos solúvel uma vez que diminui a quantidade de íons $\mathrm{Al}(\mathrm{OH})_{4}{ }^{-}$por meio da formação de $\mathrm{NaAl}(\mathrm{OH})_{4}$.

O mesmo comportamento é verificado na presença do aditivo carbonato de lítio, ou seja, a presença de $\mathrm{Li}^{+}$em solução proporciona a formação de compostos insolúveis do tipo $\mathrm{LiAl}(\mathrm{OH})_{4}$, retirando íons $\mathrm{Al}(\mathrm{OH})_{4}^{-}$da solução. A precipitação desses compostos é mais rápida do que a precipitação dos hidratos de aluminato de cálcio e apresentam estrutura cristalina necessária para servir de germes para a nucleação do hidrato $\mathrm{C}_{2} \mathrm{AH}_{8}$, acelerando a pega dos cimentos.

Os aditivos, ácido cítrico e citrato de diamônio, apresentam o maior efeito retardador por possuírem mais sítios ionizáveis e também as maiores constantes ácidas, produzindo assim maior número de ânions com grande afinidade pelo cálcio. $\mathrm{O}$ seqüestro dos íons $\mathrm{Ca}^{2+}$ pelos seus ânions diminui a relação entre as concentrações dos íons provenientes da dissolução, resultando na precipitação do hidrato mais solúvel. Tais aditivos apresentam-se também como os mais eficientes para a combinação com o acelerador $\mathrm{Li}_{2} \mathrm{CO}_{3}$ com o intuito de obter um tempo de pega que permita a trabalhabilidade do material reduzindo o tempo necessário para a sua desmoldagem.

\section{AGRADECIMENTOS}

À Almatis pelo fornecimento das matérias-primas, a SKW Polymers pelo fornecimento de aditivos e à FAPESP pelo suporte financeiro.

\section{REFERÊNCIAS}

[1] C. Parr, C. Wohrmeyer, B. Valdelievre, A. Namba, "Effect of formulation parameters upon the strength development of calcium aluminate cement containing castables", J. Techn. Assoc. Refractories 23, 4 (2003) 231-238.

[2] H. Fryda, K. Scrivener, T. Bier, B. Espinosa, "Relation between setting properties of low cement castables and interactions within the binder system (CAC-fillersadmixtures-water)", Proc. UNITECR 95 (Unified International Technical Conference on Refractories), Kyoto, Japão (1995) 1315-1323.

[3] T.A. Bier, N. E. Bunt, C. Parr, "Calcium aluminate bonded castables: their advantages and applications", Proc. The Latin-American Association of Refractory Manufacturers Meeting, Buenos Aires, Argentina (1996) 75-84.

[4] K. M. Parker, J. H. Sharp, "Refractory calcium aluminate 
cements", Trans. J. Brit. Ceram. Soc. 81 (1982) 35-42.

[5] C. M. George, "Aspects of calcium aluminate cement hydration", Refractories Symposium, The Am. Ceram. Soc., St. Louis, USA (1994) 1-21.

[6] W. Gebner, S. Mohmel, A. Sschmalstieg, A. Rettel, J. P. Bayoux, A. Capmas, A. Mathieu, "On the influence of the specific surface area and $\mathrm{Na}_{2} \mathrm{O}$ content of aluminas on the hydration processes in $\mathrm{CaO} . \mathrm{Al}_{2} \mathrm{O}_{3} / \mathrm{Al}_{2} \mathrm{O}_{3}$ Mixes", Proc. UNITECR 95 (Unified International Technical Conference on Refractories), Kyoto, Japão (1995) 313-320.

[7] C. Jolicoeur, M. A. Simard, "Chemical admixturecement interactions: phenomenology and physico-chemical concepts", Cement Concrete Composites 20 (1998) 87-101. [8] S. A. Rodger, D. D. Double, "The chemistry of hydration of high alumina cement in the presence of accelerating and retarding admixtures", Cement and Concrete Research 14 (1984) 73-82.

[9] B. R. Currell, R. Grzeskowlak, H. G. Midgley, J. R. Parsonage, "The acceleration and retardation of set high alumina cement by additives", Cement and Concrete Research 7 (1987) 420-432.

[10] N. Bunt, C. Revais, M. Vialle, "Additives in calcium aluminate cement containing castables", Proc. UNITECR 97 (Unified International Technical Conference on Refractories), New Orleans, USA (1997) 1347-1354.
[11] C. Alt, L. Wong, C. Parr, "Measuring castable rheology by exothermic profile", Refractories Appl. and News 8, 2 (2003) 15-18.

[12] G. H. Kirby, J. A. Lewis, "Comb polymer architecture effects on the rheological property evolution of concentrated cement suspensions", J. Am. Ceram. Soc. 87, 9 (2004) 1643 1652.

[13] I. R. Oliveira, J. R. Garcia, V. C. Pandolfelli, "Cinética de hidratação de ligantes a base de alumina hidratável ou aluminato de cálcio", Cerâmica 52, 323 (2005).

[14] D. Sorrentino, F. Sorrentino, M. George, "Mechanisms of hydration of calcium aluminate cements", Mater. Sci. Concrete IV, Ed. J. Skalny and S. Mindess, Am. Ceram. Soc. Westerville, $\mathrm{OH}$, USA.

[15] T. A. Bier, A. Mathieu, B. Espinosa, J. P. Bayoux, "The use of conductimetry to characterize the reactivity of calcium aluminate cements", Proc. UNITECR 93 (Unified International Technical Conference on Refractories), S. Paulo, SP, Brasil (1993) 705-716.

[16] T. A. Bier, A. Mathieu, B. Espinosa, C. Marcelon, "Admixture and their interactions with high range calcium aluminate cement", Proc. UNITECR 95 (Unified International Technical Conference on Refractories), Kyoto, Japão (1995) 357-361.

(Rec. 09/10/2005, Ac. 24/02/2006) 